\title{
ECG DENOISING AND COMPRESSION BY SPARSE 2D SEPARABLE TRANSFORM WITH OVERCOMPLETE MIXED DICTIONARIES
}

\author{
A. Ghaffari, H. Palangi, M. Babaie-Zadeh* \\ Electrical Engineering Department, \\ Sharif University of Technology, Tehran, Iran
}

\author{
C. Jutten \\ GIPSA-lab, Grenoble, France \\ and Institut Universitaire de France
}

\begin{abstract}
In this paper, an algorithm for ECG denoising and compression based on a sparse separable 2-dimensional transform for both complete and overcomplete dictionaries is studied. For overcomplete dictionary we have used the combination of two complete dictionaries. The experimental results obtained by the algorithm for both complete and overcomplete transforms are compared to soft thresholding (for denoising) and wavelet $\mathrm{db} 9 / 7$ (for compression). It is experimentally shown that the algorithm outperforms soft thresholding for about $4 d B$ or more and also outperforms Extended Kalman Smoother filtering for about $2 d B$ in higher input SNRs. The idea of the algorithm is also studied for ECG compression, however it does not result in better compression ratios than wavelet compression.
\end{abstract}

Index Terms - Sparse Representation, Sparse Decomposition, ECG Denosing, Sparse Coding, ECG Compression.

\section{INTRODUCTION}

The electrocardiographic (ECG) signals are one of the most important tools for diagnosis of cardiovascular diseases. Therefore their clarity is vital. These signals are usually contaminated by noise and therefore denoising is a crucial preprocessing step for these signals. A common approach for ECG denoising is ensemble averaging but it requires averaging many beats and in this way many important inter-beat variations are lost [1]. Another approach that can be used for ECG denoising is Wiener filtering however Wiener filter is optimal only for stationary signals. Noisy ECG has a non-stationary nature and hence Wiener filtering can't give good results for ECG denoising. Nowadays, a very common approach for denoising is wavelet denoising proposed by Donoho [2]. Also a newly efficient method mainly based on Kalman filtering is proposed in [3] which uses a nonlinear bayesian filtering framework for ECG denoising.

\footnotetext{
*This work has been partially supported by Iran NSF (INSF) under contract number 86/994, by Iran Telecommunications Research Center (ITRC), and also by ISMO and French embassy in Iran in the framework of a GundiShapour collaboration program.
}

On the other hand, off line recorded ECG signals are also necessary for telecardiology, therefore compression is also desirable if an ECG signal is to be transmitted to a cardiac specialist. Moreover there may exist a necessity to build a database of ECG recordings for patients so that two ECG traces taken on different dates may be compared.

Schemes of ECG data compression are grouped into two categories: time domain (direct) methods and transform methods (see [4], [5], [6]). In direct methods, the compression is performed directly on the ECG samples but in transform methods signal is transformed to another domain in which signal is sparsely represented.

In this article a strategy based on an enhanced sparse representation in transform domain (for both complete and overcomplete dictionaries) for ECG denoising and compression is studied which is based on a recently proposed approach [7] for image denoising and also a recently proposed two dimensional sparse decomposition algorithm [8]. An enhanced sparse representation can be achieved by grouping similar 1D segments of the input signal into 2D data arrays. We have used this approach with a $2 D$ separable complete and overcomplete dictionary (DCT+Wavelet or overcomplete $D C T)$ for ECG denoising and compression. Note that to use the approach proposed in [8], separability of dictionary is an essential assumption. Our procedure includes three steps: 2D transformation using the dictionary (complete or overcomplete $)^{1}$, shrinkage of the transform domain coefficients, and inverse 2D transformation. Due to the similarity between segments in a 2D array, the 2D transform can achieve a highly sparse representation. Experimental results demonstrate that its performance is highly better than Wavelet based denoising proposed in [2] and also better than extended Kalman smoother filtering proposed in [3](for higher input SNRs) but it does not achieve outstanding performance (compared to Wavelet) for ECG compression in terms of both SNR and sparsity.

The paper is organized as follows. The next section describes the main idea and discusses its effectiveness. Section

\footnotetext{
${ }^{1}$ Note that the initial data are one dimensional and we arrange it in two dimensional data before applying the $2 \mathrm{D}$ transform. In fact, this step is the most important step in our algorithm.
} 
3 introduces two dimensional sparse decomposition based on [8]. The algorithm is then stated in Section 4. Finally, Section 5 provides some experimental results of algorithm.

\section{THE BASIC IDEA}

The basic idea of this article is achieving an enhanced sparse representation by grouping similar segments of the input ECG signal into 2D arrays, and then using a 2D transformation (which can be complete or overcomplete) to transform 2D arrays.

A simple justification for the effectiveness of the idea is as follow:

- Assume that the grouping is done, i.e. similar segments are placed in groups and a 1D transformation (A) is used for each group.

- In each group we have similar segments and hence after transformation we will have the same number of highmagnitude coefficients for each segment in a group, say $\alpha$ high-magnitude coefficients for each segment.

- Assuming $n$ segments in each group, we will have $n \alpha$ high-magnitude coefficients in that group. In other words this group can be represented by $n \alpha$ coefficients.

- Now we should perform another 1D transform (B) on the second dimension (along each row) of each group.

- Components of this row are similar (because only similar segments are in this group), i.e. there is a kind of similarity for all members of the row.

- As an example, after using 1D transform the first or second coefficients of this row will be high-magnitude (because of the compaction property of used transform). This means that the whole group can be represented by highly less than $n \alpha$ coefficients instead of $n \alpha$ coefficients (i.e., a much more sparse representation).

Note that if overcomplete 2D transform be used we will have an underdetermined system of linear equations and we have to find its sparsest solution. Also note that we have grouped various segments of 1D ECG signal into some two dimensional groups, hence we are working with two dimensional data and working with a fast and less complex algorithm to find the sparsest solution is essential. To do this we use 2DSL0 algorithm [8], as a fast method to obtain the sparsest solution of an underdetermined system of linear equations. Therefore before presenting the algorithm two dimensional sparse decomposition is briefly described in the next section.

\section{SPARSE DECOMPOSITION OF TWO DIMENSIONAL SIGNALS}

In signal decomposition, a signal vector $\mathrm{x} \in \mathbb{R}^{n}$ is to be decomposed as a linear combination of $m$ basic signals $\boldsymbol{\varphi}_{i} \in \mathbb{R}^{n}, 1 \leq i \leq m$, This can be written as $\mathbf{x}=$ $s_{1} \boldsymbol{\varphi}_{1}+\cdots+s_{m} \boldsymbol{\varphi}_{m}=\boldsymbol{\Phi} \mathbf{s}$, where $\boldsymbol{\Phi}=\left[\boldsymbol{\varphi}_{1}, \ldots, \boldsymbol{\varphi}_{m}\right]$ and $\mathbf{s}=\left(s_{1}, \ldots, s_{m}\right)^{T}$. The signals $\boldsymbol{\varphi}_{i}, i=1, \ldots, m$ are called 'atoms', and they collectively form the 'dictionary' $\boldsymbol{\Phi}$. When the dictionary is overcomplete, i.e. when $m>n$, the decomposition is not unique, and the goal of sparse decomposition (SD) is then to search for a decomposition in which as few as possible atoms are present in the decomposition. Mathematically, this is equivalent to finding the sparsest solution of the Underdetermined System of Linear Equations (USLE) $\Phi \mathbf{s}=\mathbf{x}$.

Now consider the decomposition of two-dimensional (2D) signals: $\mathbf{X} \in \mathbb{R}^{n_{1} \times n_{2}}$ is to be decomposed as linear combination of atoms $\boldsymbol{\Phi}_{i j}, 1 \leq i \leq m_{1}, 1 \leq j \leq m_{2}$, that is $\mathbf{X}=\sum_{i=1}^{m_{1}} \sum_{j=1}^{m_{2}} s_{i j} \boldsymbol{\Phi}_{i j}$. Letting $\mathbf{x}=\operatorname{vec}(\mathbf{X})$ and $\boldsymbol{\varphi}_{i j}=\operatorname{vec}\left(\boldsymbol{\Phi}_{i j}\right)$, where 'vec' of a matrix stands for the vector obtained by stacking its columns, this $2 \mathrm{D}$ problem will be converted to the previous 1D problem. However, the atoms usually used in 2D signal transformation and representation (e.g. 2D Fourier atoms) are separable, that is, there are vectors $\mathbf{a}_{i}$ and $\mathbf{b}_{j}$ such that $\boldsymbol{\Phi}_{i j}=\mathbf{a}_{i} \mathbf{b}_{j}^{T}, 1 \leq i \leq m_{1}, 1 \leq j \leq m_{2}$. This kind of atoms result in 2D decompositions (and transformations) of the form:

$$
\mathbf{X}_{n_{1} \times n_{2}}=\mathbf{A} \mathbf{S}_{m_{1} \times m_{2}} \mathbf{B}^{T}
$$

where $\mathbf{A}=\left[\mathbf{a}_{1}, \ldots, \mathbf{a}_{m_{1}}\right]$ and $\mathbf{B}=\left[\mathbf{b}_{1}, \ldots, \mathbf{b}_{m_{2}}\right]$. For the complete case, i.e. where $n_{1}=m_{1}$ and $n_{2}=m_{2}$, the above decomposition is unique. However, for the overcomplete case, i.e. where $n_{1}<m_{1}$ and $n_{2}<m_{2}$, the representation is not unique, and in Sparse Decomposition (SD), it is desired to find the $\mathbf{S}$ with as much zero elements as possible. Note that in our simulations $\boldsymbol{B}$ is always a complete Haar dictionary for denoising and a complete DCT dictionary for compression and $\boldsymbol{A}$ is either a complete DCT, or a complete Wavelet, or an overcomplete mixed DCT-Wavelet.

It can be shown $[9,10,11]$ that if the USLE $\mathbf{\Phi} \mathbf{s}=\mathbf{x}$ has a solution for which $\|\mathbf{s}\|_{0}<\operatorname{spark}(\boldsymbol{\Phi}) / 2$, it is the unique sparsest solution $^{2}$. In [8] the uniqueness conditions for the two dimensional case are extracted (which are less restrictive) conditions than those of $[9,10,11])$. Moreover a fast algorithm is presented (in [8]) which is called two dimensional smoothed $\ell^{0}$ (2DSL0) algorithm. This algorithm will be used in this paper to find the sparsest solution when we have an overcomplete mixed dictionary.

\footnotetext{
${ }^{2}$ spark of a matrix [10] stand for the minimal number of its columns which are linearly dependent
} 


\section{THE ALGORITHM}

Based on the basic idea of the previous section, the final algorithm is as presented in Fig.1.

Remark 1. In the matching phase (which is used for grouping the segments) we are looking for segments that are similar to a reference segment. It needs a search among all segments to find segments similar to a given reference segment. The fragments whose distance from the reference segment is smaller than a grouping threshold are stacked in a group. Any signal fragment can be used as a reference segment and thus a group can be constructed for it. The similarity between signal fragments is typically computed as the inverse of some distance measure. In particular, we use the same distance proposed in [7] which is defined below:

$$
d\left(\mathbf{y}_{\mathbf{r}}, \mathbf{y}_{\mathbf{i}}\right)=\frac{\left\|\mathbf{y}_{\mathbf{r}}-\mathbf{y}_{\mathbf{i}}\right\|_{2}^{2}}{N}
$$

where, $\mathbf{y}_{\mathbf{r}}$ is the reference segment from which the distance of the $i^{\text {th }}$ segment $\left(\mathbf{y}_{\mathbf{i}}\right)$ is calculated. $N$ is the size of the chosen segments (for all our simulations segments with 32 samples are used). This distance can also be computed in the transform domain, i.e., we can do the grouping after 1D transformation (transform domain grouping). Our experience with the algorithm (not presented in this paper) shows that there is no significant change in results.

Remark 2. Note that in the $2 \mathrm{D}$ transformation we can have complete or overcomplete transform. If a complete transform is used the procedure of algorithm is straightforward (using $\ell^{2}$ norm). But if an overcomplete 2D transform is used we will have an underdetermined system of linear equations and we have to find its sparsest solution. To do this we use 2DSL0 algorithm [8], as a fast method to obtain the sparsest solution of an underdetermined system of linear equations.

Remark 3. Based on (1) the overcomplete 2D transform has been constructed from an overcomplete 2D dictionary (A) which is a concatenation of two complete dictionaries and another complete dictionary $(\mathbf{B})^{3}$. Note that the overcompleteness factor (number of columns of dictionary divided by number of rows of dictionary) for this dictionary is 2 .

Remark 4. As it is seen in Fig. 1, in the shrinkage phase we have used a hard thresholding methodology.

Remark 5. Obviously, a straightforward implementation of this algorithm is highly computationally demanding. In order to realize a practical and efficient algorithm, we can, for example, reduce the number of

\footnotetext{
${ }^{3}$ Explanations regarding choosing these dictionaries are presented in simulation results.
}

- Grouping:

1. Segment input signal to approximately 0.25 seconds time intervals $^{a}$

Denoising: In this case number of overlapping samples between two adjacent segments is 31 samples.

Compression: In this case there are at most 12 overlapping samples.

2. Save segments in $\mathbf{Y}$.

- While $\mathbf{y}$ is not empty

for $\mathrm{i}=1, \ldots$, NumberOfSegments:

1. Choose one segment as a reference segment $\left(\mathbf{y}_{\mathbf{r}}\right)$.

2. Calculate $d\left(\mathbf{y}_{\mathbf{r}}, \mathbf{y}_{\mathbf{i}}\right)=\frac{\left\|\mathbf{y}_{\mathbf{r}}-\mathbf{y}_{\mathbf{i}}\right\|_{2}^{2}}{N}$ were $\mathbf{y}_{\mathbf{i}}$ is the $i^{\text {th }}$ segment.

3. if $d\left(\mathbf{y}_{\mathbf{r}}, \mathbf{y}_{\mathbf{i}}\right) \leq$ ThresholdDistance

- Assign $\mathbf{y}_{\mathbf{i}}$ to the current group.

- Remove $\mathbf{y}_{\mathbf{i}}$ from $\mathbf{Y}$.

Save resulted group in a 2D array named GroupArray

- 2D transform

- If Complete 2D transform is desired:

1. For every group of GroupArray

In (1) set $\mathbf{A}$ as a complete dictionary and set $\mathbf{B}$ as another complete dictionary.

Solve determined system of linear equations (straightforward using $\ell^{2}$ norm)

- If Overcomplete 2D transform is desired:

1. For every group of GroupArray

In (1) set $\mathbf{A}$ as an overcomplete dictionary and set $\mathbf{B}$ as a complete dictionary.

Use 2DSL0 algorithm to solve under determined system of linear equations

- Shrinkage

If TransformDomainCoefficients $\leq$ HardThreshold

Discard that coefficient

- Calculate inverse 2D transform

- For complete transform:

Set $\mathbf{A}$ as complete dictionary and set $\mathbf{B}$ as a complete dictionary too.

- For overcomplete transform:

In (1) set $\mathbf{A}$ as an overcomplete dictionary and set $\mathbf{B}$ as a complete dictionary.

- Place each decoded segment in its original position.

${ }^{a}$ based on sampling rate of ECG signals which we have used each of these fragments includes 32 samples

Fig. 1. The Algorithm 
processed segments using only a limited number of reference segments by choosing reference segments between every $N_{1}$ segments. In this way we will have (TotalNumberOfSegments) $/ N_{1}$ reference segments. A complete set of such ideas can be found in [7].

Remark 6. Note that in the application of this approach for denoising, the blocks have an overlapping of 31 samples which results in high redundancy (in simulation results we will see that this redundancy is one of the main reasons for high performance of the proposed algorithm). But in compression applications we cannot use this redundancy as well because accompanying with an acceptable SNR there should be an acceptable compression ratio too and if we use much redundancy (too much overlapping samples), we will have good SNR but compression ratio will degrade.

Remark 7. Reasons for expecting good results especially in denoising are as follow:

- Because of the redundancy between various segments there will be more than one estimation for each sample and the final value of that sample is a weighted average of its estimations.

- Aforementioned redundancy results in a very good matching in each group and hence an enhanced sparse representation.

\section{SIMULATION RESULTS}

In this section, we study the performance of the presented approach in overcomplete and complete cases. This section includes two parts. In the first part, the performance of the proposed approach is investigated in denoising application. In the second part, its performance is investigated for compression. The MIT-BIH Normal Sinus Rhythm Database [12],[13] was used to study the performance of the proposed approach. In our simulations ten ECG signals were randomly selected from eighteen ECG signals (from database in [13]) and then from each ECG signal a 2500 samples segment was used as the test signal. The final results are average results of these ten signals.

\subsection{Denoising}

The additive noise is assumed to be white Gaussian noise. Input SNR (i.e. signal to noise ratio between original signal and noisy signal) and output $\mathrm{SNR}^{4}$ (i.e. signal to noise ratio

\footnotetext{
${ }^{4}$ The definition of SNR which has been used here is $10 \log \left(\frac{\|\mathbf{x}\|_{2}^{2}}{\|\mathbf{n}\|_{2}^{2}}\right)$ as input SNR and $10 \log \left(\frac{\|\mathbf{x}\|_{2}^{2}}{\left\|\mathbf{x}-\mathbf{x}_{\mathbf{r}}\right\|_{2}^{2}}\right)$ as output SNR. In these formulations $\mathbf{x}$ is the original signal, $\mathbf{n}$ is noise and $\mathbf{x}_{\mathbf{r}}$ is the reconstructed signal
}

between original signal and reconstructed signal) were calculated for some denoising methods. The methodologies compared in our simulations are soft thresholding [2], Extended Kalman Smoother filtering [3], 2D denoising based on complete DCT dictionary (proposed in this paper), 2D denoising based on complete Wavelet dictionary (proposed in this paper) and 2D denoising based on overcomplete DCT+Wavelet dictionary (proposed in this paper). Note that the $\mathbf{B}$ in (1) is assumed to be Haar transform. Results are presented in Fig. 2. As it can be seen from this figure results of denoising using proposed $2 \mathrm{D}$ dictionaries are about $4 \mathrm{~dB}$ better than denoising using soft thresholding and about $2 d B$ better than extended Kalman smoother filtering for higher input SNRs. Among 2D dictionaries the best results are obtained with 2D complete Wavelet dictionary.

\subsection{Compression}

Ten ECG signals with 2500 samples were used for experiments of this part. For compression we have at most 12 samples overlap. In fact based on the matching criteria defined in (2) the best matching block will be chosen between these overlapping blocks. Five methodologies are compared in this part, Wavelet db9/7, complete DCT dictionary, complete Wavelet dictionary, overcomplete mixed (DCT+Wavelet) dictionary and overcomplete DCT dictionary. The overcomplete 2D DCT transform has been constructed from an overcomplete 2D DCT transform defined as (3).

$\phi_{k}(n)=\cos \left(\frac{k \pi(n+0.5)}{M}\right), n \in 0, \ldots, N-1, k \in 0, \ldots, M-1$

In our simulations we have assumed that overcompleteness factor is 2. The results are presented in Fig.3.

\section{CONCLUSION}

In this paper an idea for ECG denoising and compression using complete and overcomplete dictionaries was studied. The idea is based on 2D transform (complete or overcomplete) to enhance the sparsity of the coefficients. Our simulations show that the usage of this idea (for both complete and overcomplete cases) enhances the denoising results compared to soft thresholding about $4 d B$ and extended Kalman smoother filtering about $2 d B$ for higher input SNRs, but it does not give outstanding results for ECG signal compression.

\section{ACKNOWLEDGEMENT}

The authors would like to thank Dr. Sameni (Shiraz university, Shiraz, Iran) to provide us with their toolbox for Extended Kalman Smoother method in ECG denoising. 


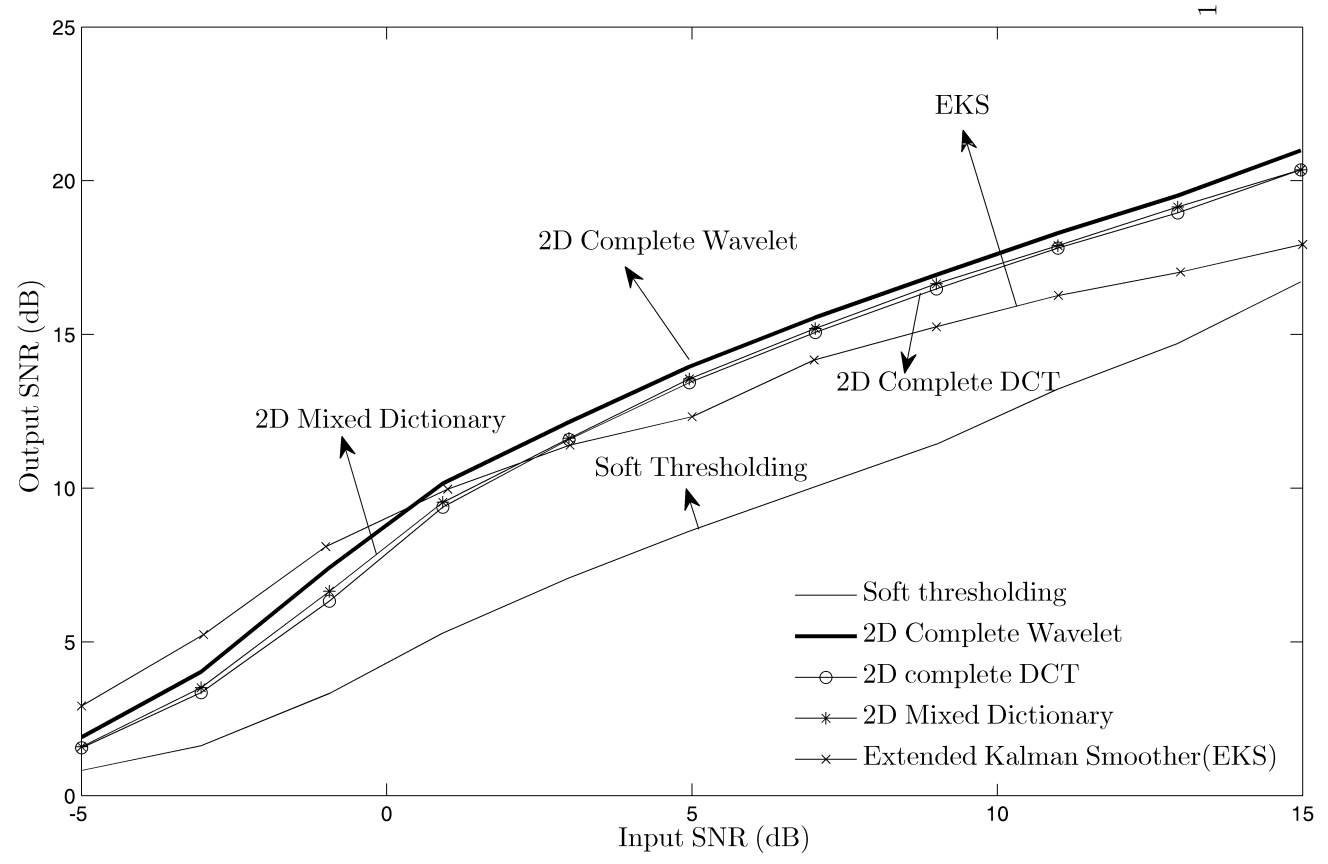

Fig. 2. Comparison of performance of various denoising methodologies

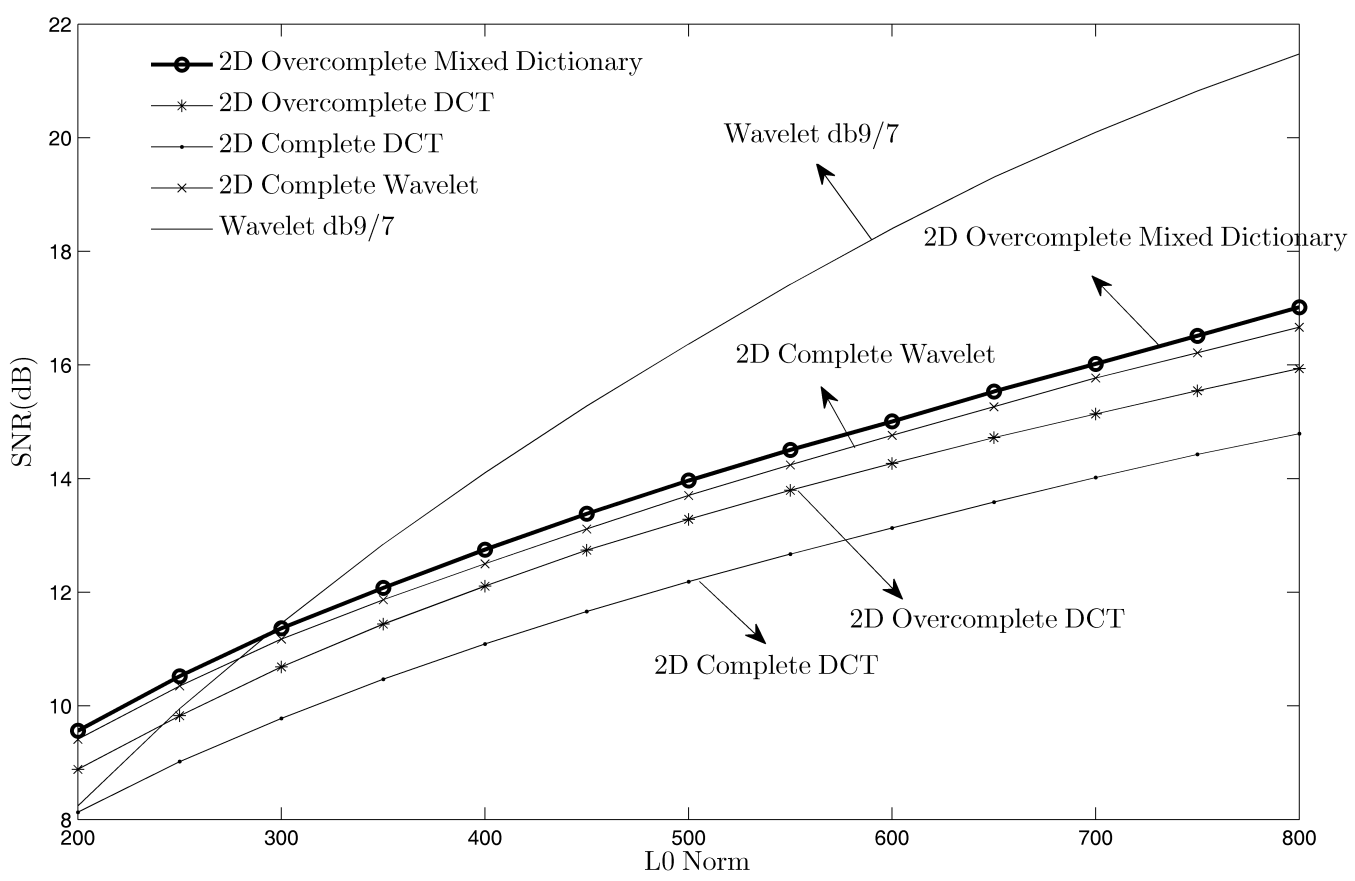

Fig. 3. Comparison of performance of various Compression methodologies 


\section{REFERENCES}

[1] P. Lander and E. J. Berbari, “Time frequency plane wiener filtering of the high resolution ecg: development and applications," IEEE Trans. on Biomedical Engineering, vol. 44, pp. 256-265, 1997.

[2] D. L. Donoho, "Denoising by soft-thresholding," IEEE Trans. on Information Theory, vol. 41, pp. 613-627, 1995.

[3] R. Sameni, M. B. Shamsollahi, C. Jutten, and G. D. Clifford, "A nonlinear Bayesian filtering framework for ECG denoising," IEEE Trans. on Biomedical Engineering, vol. 54, pp. 2172-2185, 2007.

[4] J. Chen and S. Itoh, "A wavelet transform-based ecg compression method guaranteeing desired signal quality," IEEE Trans. on Biomedical Engineering, vol. 45, no. 12, pp. 1414-1419, 1998.

[5] M. Hilton, "Wavelet and wavelet packet compression of electrocardiograms," IEEE Trans. on Biomedical Engineering, vol. 44, pp. 394-402, 1997.

[6] S. Jalaleddine, C. Hutchens, R. Strattan, and W. Cobberly, "Ecg data compression tecniques-a unified approach," IEEE Trans. on Biomedical Engineering, vol. 37, pp. 329-343, 1990.

[7] K. Dabov, A. Foi, V. Katkovnik, and K. Egiazarian, "Image denoising by sparse 3D transform-domain collaborative filtering," IEEE Trans. on Image Processing, vol. 16, no. 8, pp. 2080-2095, 2007.

[8] A. Ghaffari, M. Babaie-Zadeh, and C. Jutten, "Sparse decomposition of two dimensional signals," accepted in ICASSP09, Taiwan, 2009.

[9] I. F. Gorodnitsky and B. D. Rao, "Sparse signal reconstruction from limited data using focuss, a reweighted minimum norm algorithm," IEEE Transactions on Signal Processing, vol. 45, no. 3, pp. 600-616, March 1997.

[10] D. L. Donoho and M. Elad, "Maximal sparsity representation via $\ell^{1}$ minimization," the Proc. Nat. Aca. Sci., vol. 100, no. 5, pp. 2197-2202, March 2003.

[11] R. Gribonval and M. Nielsen, "Sparse decompositions in unions of bases," IEEE Trans. Inform. Theory, vol. 49, no. 12 , pp. 3320-3325, Dec. 2003.

[12] L. Glass J. M. Hausdorff P. C. Ivanov R. G. Mark J. E. Mietus G. B. Moody C.-K. Peng A. L. Goldberger, L. A. N. Amaral and H. E. Stanley, "Physiobank, physiotoolkit, and physionet: Components of a new research resource for complex physiologic signals," Circulation, vol. 101, no. 23, pp. 215-220, June 2000.
[13] The MIT-BIH Normal Sinus Rhythm Database., url=http: / / www . physionet . org/ physiobank/database/nsrdb/. 